\title{
An Evaluation of Internal Combustion Engines as the Prime Movers in CHP Systems
}

\author{
Mehdi Aghaei Meybodi and Masud Behnia* \\ School of Aerospace, Mechanical and Mechatronic Engineering, The University of Sydney, NSW 2006, Australia \\ *Corresponding author.Tel: +61290369518,E-mail:m.behnia@usyd.edu.au
}

\begin{abstract}
Optimum selection of prime movers in combined heat and power (CHP) systems is of crucial importance due to the fact that inappropriate choices reduce the benefits of CHP systems considerably. In the selection procedure the performance characteristics of prime movers as well as economic parameters should be considered. The aim of this paper is to present a thermo-economic approach to selecting the optimum nominal power and planning the operational strategy of internal combustion engines in a medium scale combined heat and power system is presented using the Net Annual Cost (NAC) criterion. Three modes of operation have been considered, namely one-way connection (OWC) mode, two-way connection (TWC) mode, and heat demand following (HDF) mode. The proposed method has been used for a case study. It has been observed that the optimum nominal powers in the case of using gas engines are 3.3 MW, 3.2 MW, and 1.2 MW and in the case of using diesel engines are 3.4 MW, 3.4 MW, and 1.4 MW for TWC, OWC, and HDF modes respectively. The proposed method may be also used for other types of prime movers as well as various sizes of combined heat and power systems.
\end{abstract}

Keywords: Combined Heat and Power System, Internal Combustion Engines.

\section{Nomenclature}

\begin{tabular}{|c|c|}
\hline$A C T$ & annual carbon tax. \\
\hline$C$ & cost.. \\
\hline$C C$ & capital cost. \\
\hline $\mathrm{COF}$ & cost of fuel per hour \\
\hline$\dot{H}$ & 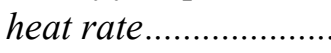 \\
\hline$H R B$ & heat recovery boiler \\
\hline $\begin{array}{l}i \\
k\end{array}$ & $\begin{array}{l}\text { interest rate................... } \\
\text { number of equipment }\end{array}$ \\
\hline
\end{tabular}

\begin{tabular}{|c|c|}
\hline 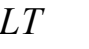 & lifetime. \\
\hline$M C$ & maintenance cost per hour \\
\hline$N$ & number of time intervals \\
\hline$N A C$ & Net Annual Cost ............... \\
\hline$P$ & electric power ............................. $\mathrm{kW}$ \\
\hline$S V$ & 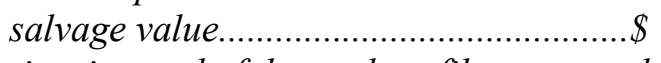 \\
\hline
\end{tabular}

\section{Subscripts}

$\begin{array}{ll}b & \text { buying electricity } \\ \text { el } & \text { electricity } \\ f & \text { fuel } \\ h & \text { heat } \\ r & \text { required } \\ s & \text { selling electricity }\end{array}$

\section{Introduction}

Combined heat and power (CHP) systems are considered to be one of the most important ways to consume the fossil fuels efficiently. These systems emit less pollution compared to the separate production of the same amount of electricity and heat. This paper presents a thermo-economic method for optimum sizing of internal combustion engines to serve as the prime movers of medium scale (500 kW-5,000 kW) combined heat and power systems. Three modes of operation: one-way connection (OWC) mode, two-way connection (TWC) mode, and heat demand following (HDF) mode are examined. In all three modes, buying electricity from the grid is possible while selling electricity to the grid is allowable in TWC and HDF modes. The objective of HDF mode is to minimize the waste of energy; therefore the engines 
work in a condition at which the excess generated heat is minimal. A backup boiler can be installed to supply heat in all three modes.

\section{Methodology}

The applied method is one of the standard engineering economy approaches which is called annual cash flow (ACF) analysis. In ACF method, all costs and benefits are converted to equivalent uniform annual cost (EUAC) and equivalent uniform annual benefit (EUAB). To compare the various options, the difference of EUAC and EUAB (EUAC-EUAB) is calculated and the one which results in the minimum value is the most economical choice [1]. Based on the annual cash flow method, the proposed objective function (Net Annual Cost (NAC) \$/year) is defined as:

$$
\begin{aligned}
& \mathrm{NAC}=\left[\sum_{j=1}^{k}\left(C C_{j}-S V_{j}\left(\frac{1}{(1+i)^{L T}}\right)\right)\right]\left(\frac{i(1+i)^{L T}}{(1+i)^{L T}-1}\right)+\mathrm{ACT}+\sum_{m=1}^{N}\left[\sum_{j=1}^{k}\left(M C_{j}+C O F_{j}\right)+P_{b} \times\right. \\
& \left.C_{e l, b}-P_{C H P, r} \times C_{e l}-\dot{H}_{C H P, r} \times C_{h}-P_{C H P, s} \times C_{e l, s}\right]_{m} \times \tau_{m}
\end{aligned}
$$

\subsection{Selection and planning of operational strategy}

To determine the optimum nominal power and the operational strategy of internal combustion engines as prime movers of CHP systems based on the specific electricity and heat demand profile, the following procedure is adopted:

- For nominal powers from $500 \mathrm{~kW}$ to $5,000 \mathrm{~kW}$ and for each time interval of demand profile $\left(\tau_{m}\right)$ with changing of partial load from $20 \%$ to $100 \%$.

- Calculating NAC for a given partial load (this is represented by $\mathrm{NAC}_{\mathrm{m}}$ ).

- Comparing the calculated $\mathrm{NAC}_{\mathrm{m}}$ values and choosing and saving the minimum value $\left(\mathrm{NAC}_{\mathrm{m}, \mathrm{min}}\right)$ and its associated partial load.

- Calculating NAC by adding up the $\mathrm{NAC}_{\mathrm{m}, \text { min }}$ values for all time intervals.

- Choosing the nominal power which results in the minimum NAC as the prime mover's nominal power of the CHP system.

- For the selected nominal power and for each time interval the partial load at which the $\mathrm{NAC}_{\mathrm{m} \text {, min }}$ is obtained is the operational strategy of CHP system.

\section{Results}

To illustrate the Net Annual Cost method, we have chosen a case study. It is noted that adoption of the specific operational mode directly depends on the method of connection to the grid which determines the possibility of selling electricity. Fig. 1 shows the electricity and heat demand profile for the case study based on operational data of a local educational institute [2].

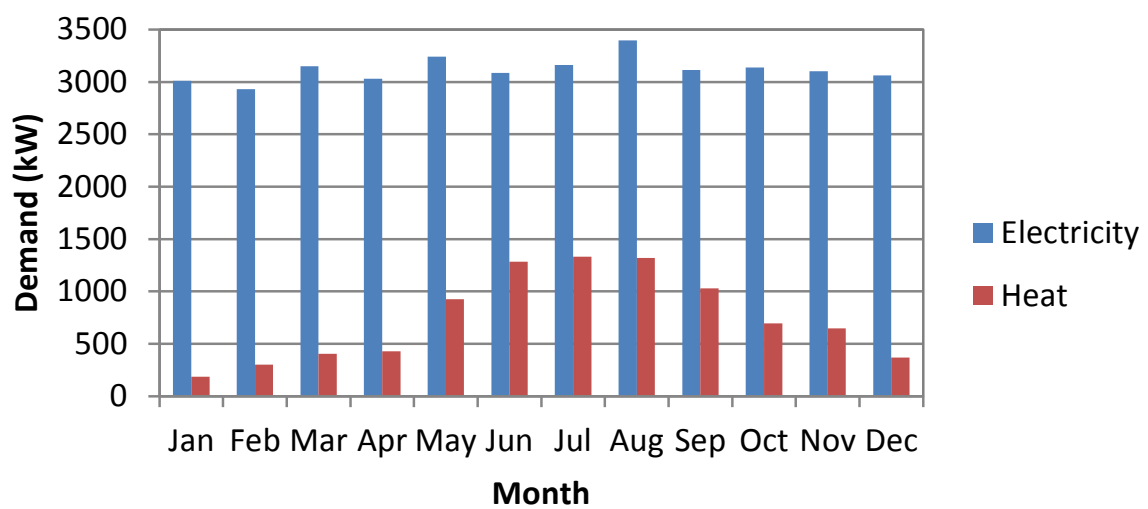

Fig 1. Demand profile for the case study 
Table 1 lists the costs of natural gas, diesel fuel, and electricity as well as the value of generated electricity and heat [3-5]. A nominal Carbon tax of $\$ 30$ per emitted tonne of $\mathrm{CO}_{2}$ equivalent is assumed [6].

Table 1. Price list

\begin{tabular}{lc}
\hline \multicolumn{1}{c}{ Items } & Price $(\$ / \mathrm{kWh})$ \\
\hline Buying electricity $\left(\mathrm{C}_{\mathrm{el}, \mathrm{b}}\right)$ & 0.18 \\
\hline Natural gas & 0.045 \\
\hline Diesel fuel & 0.117 \\
\hline Selling electricity $\left(\mathrm{C}_{\mathrm{el}, \mathrm{s}}\right)$ & 0.15 \\
\hline Generated electricity $\left(\mathrm{C}_{\mathrm{el}}\right)$ & 0.18 \\
\hline Generated heat ( gas engine) $\left(\mathrm{C}_{\mathrm{h}}\right)$ & 0.05 \\
\hline Generated heat ( diesel engine) $\left(\mathrm{C}_{\mathrm{h}}\right)$ & 0.138 \\
\hline
\end{tabular}

Figs. 2-4 illustrate the variation of Net Annual Cost versus nominal power of gas engine in three modes of operation. In TWC mode a 3.3 MW gas engine, in OWC mode a 3.2 MW gas engine, and in HDF mode a 1.2 MW gas engine have resulted in the minimum NAC values.

Fig. 2 Variation of Net Annual Cost versus gas engine nominal power in TWC mode

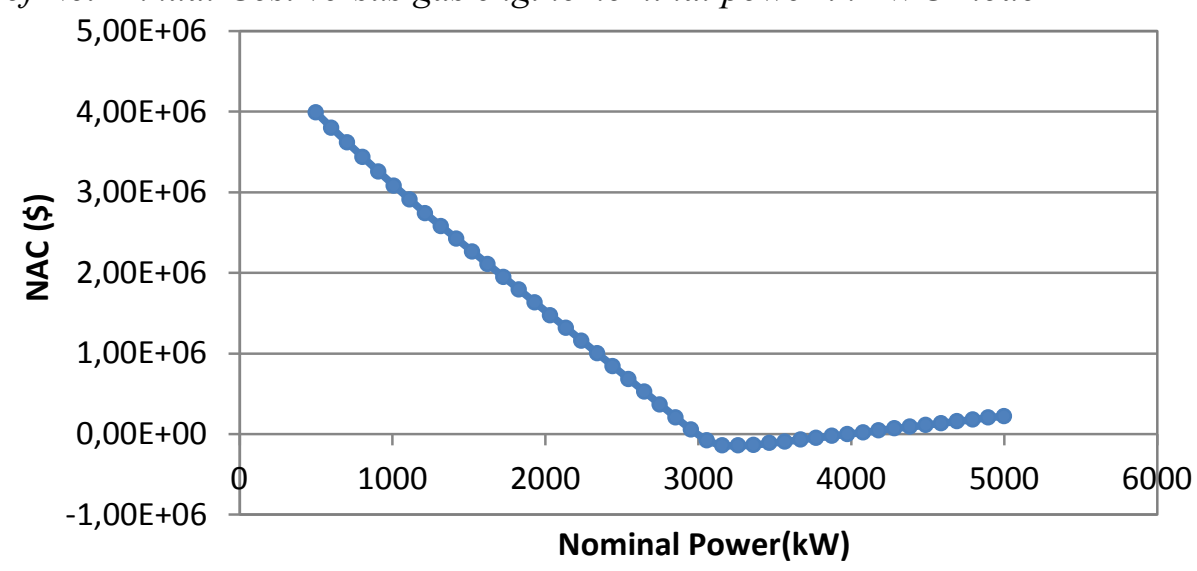

Fig. 3 Variation of Net Annual Cost versus gas engine nominal power in OWC mode

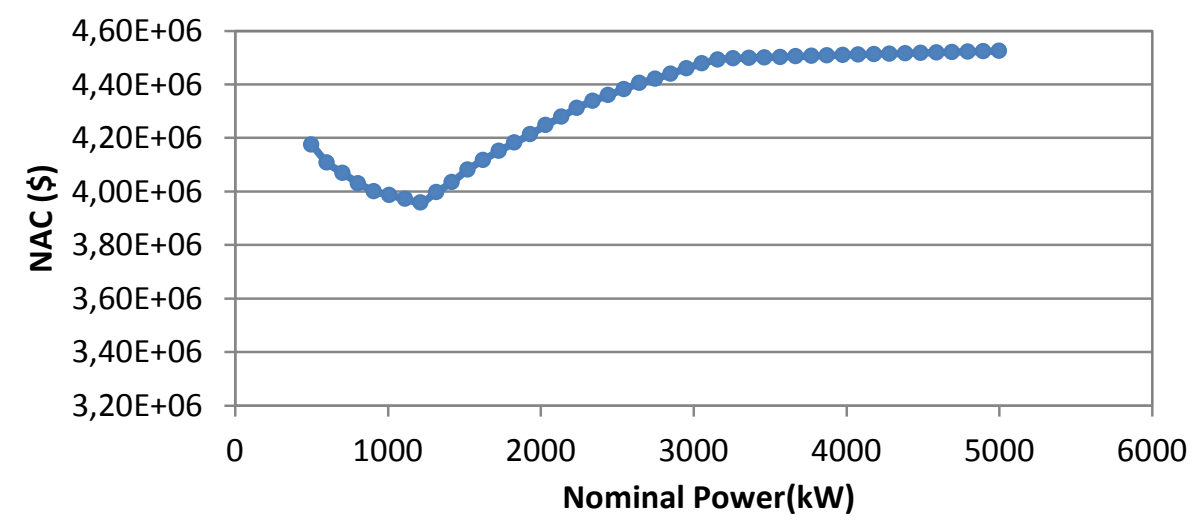

Fig. 4 Variation of Net Annual Cost versus gas engine nominal power in HDF mode

The variation of NAC with nominal power of diesel engine for three modes of operation is shown in Figs. 5-7. The optimum nominal powers in TWC, OWC, and HDF modes are 3.4 MW, 3.4 MW, and 1.4 MW, respectively. 


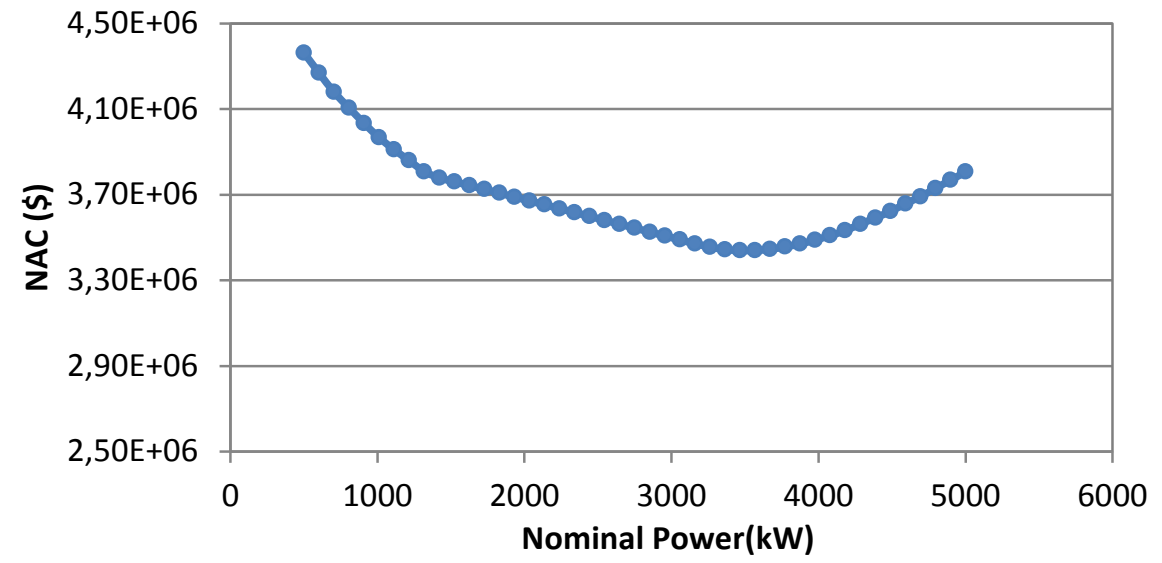

Fig. 5 Variation of Net Annual Cost versus diesel engine nominal power in TWC mode

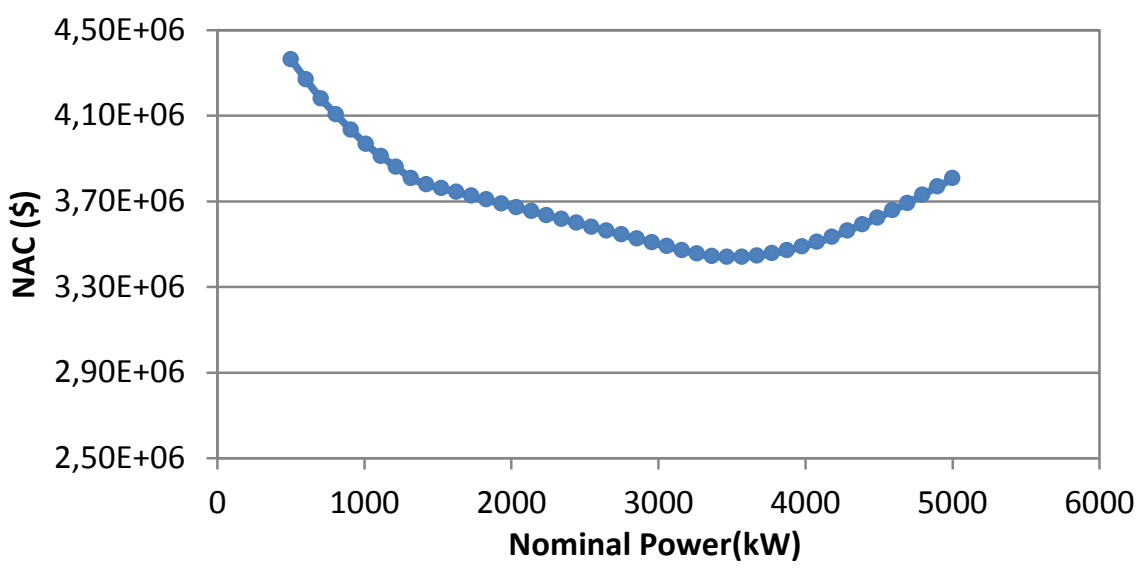

Fig. 6 Variation of Net Annual Cost versus diesel engine nominal power in OWC mode

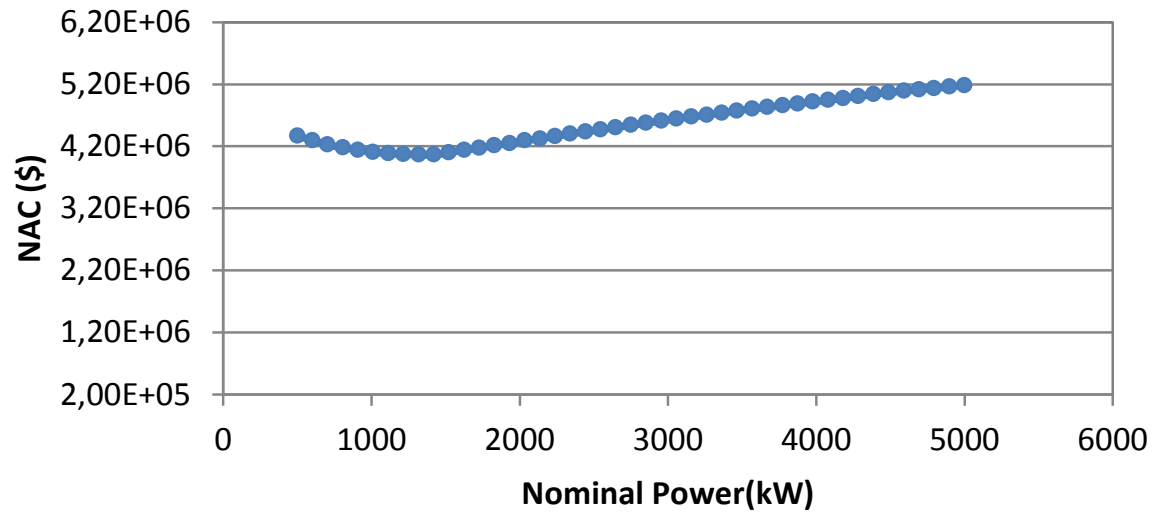

Fig. 7 Variation of Net Annual Cost versus diesel engine nominal power in HDF mode

The operational strategy of selected gas engines and diesel engines in the three modes of operation is shown in Figs. 8-13. 


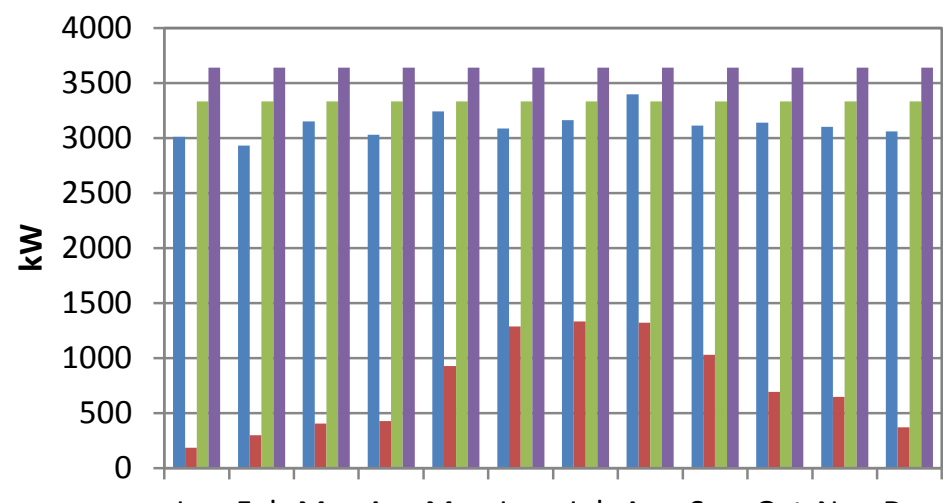

Required Elec.

Required Heat

Generated Elec.

Generated Heat

Jan Feb Mar Apr May Jun Jul Aug Sep Oct Nov Dec

Month

Fig. 8 The operational strategy of selected gas engine in TWC mode of operation

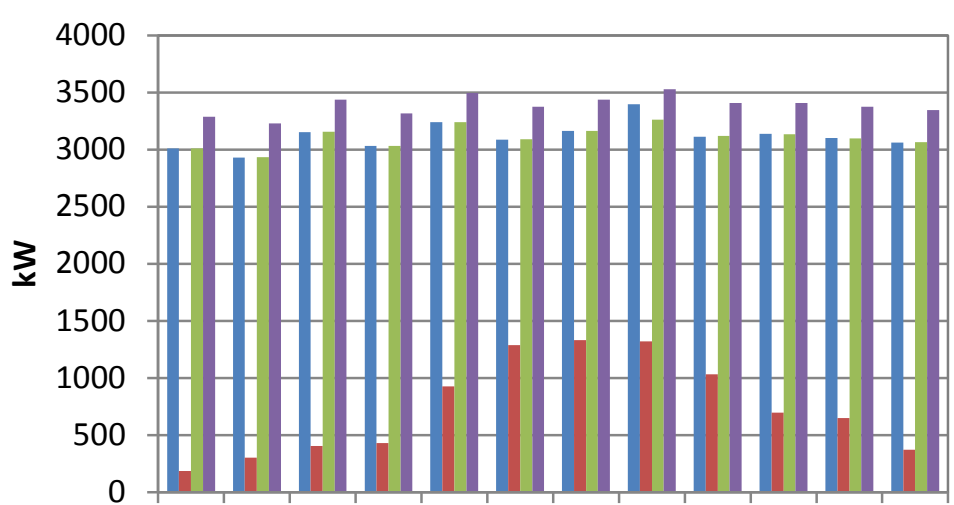

- Required Elec.

- Required Heat

- Generated Elec.

- Generated Heat

Jan Feb Mar Apr May Jun Jul Aug Sep Oct Nov Dec

Month

Fig. 9 The operational strategy of selected gas engine in OWC mode of operation

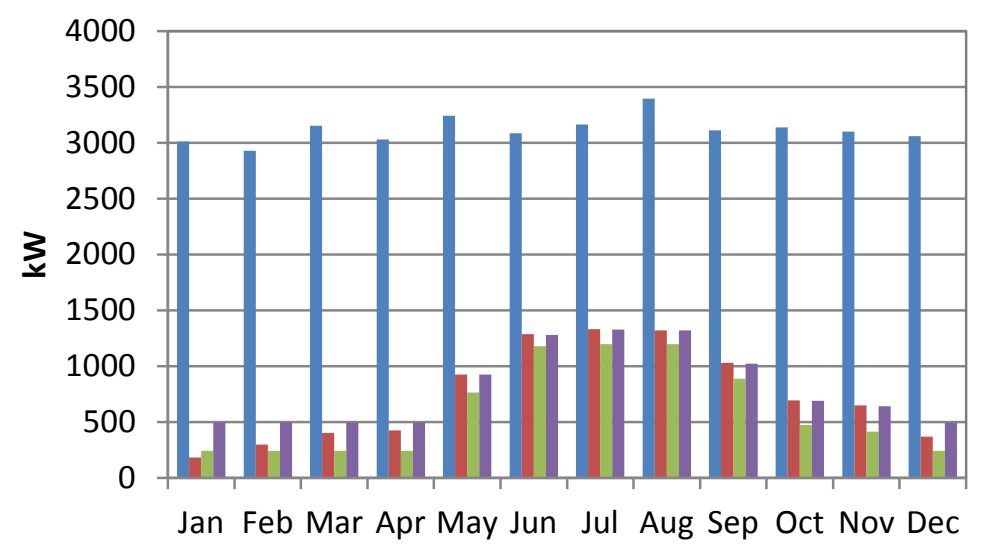

Required Elec.

Required Heat

- Generated elec.

- Generated Heat

Month

Fig. 10 The operational strategy of selected gas engine in HDF mode of operation 


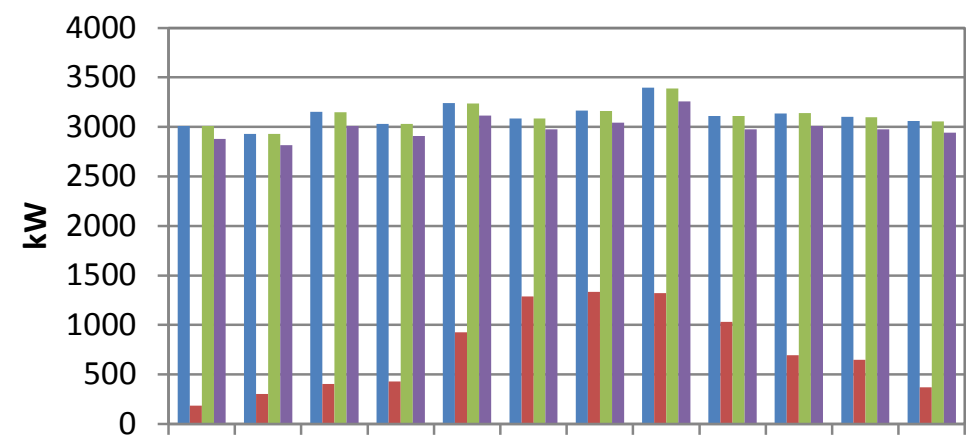

Required Elec.

Required Heat

Gnerated Elec.

Jan Feb Mar Apr May Jun Jul Aug Sep Oct Nov Dec

Generated Heat

Month

Fig. 11 The operational strategy of selected diesel engine in TWC mode of operation

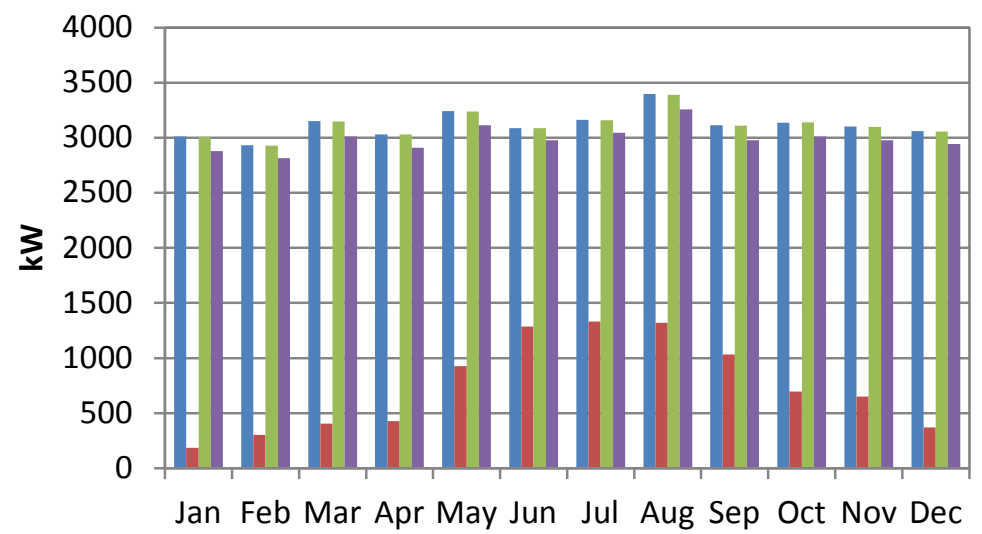

- Required Elec.

Required Heat

Gnerated Elec.

Generated Heat

Month

Fig. 12 The operational strategy of selected diesel engine in OWC mode of operation

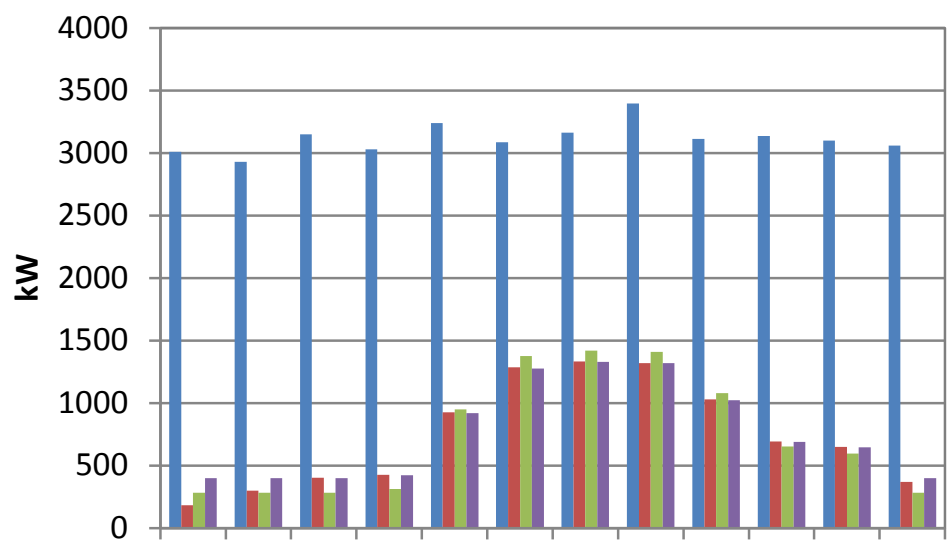

Required Elec.

Required Heat

Generated Elec.

Generated Heat

Jan Feb Mar Apr May Jun Jul Aug Sep Oct Nov Dec

Month

Fig. 13 The operational strategy of selected diesel engine in HDF mode of operation 


\section{Discussion}

The figures for the operational strategy of selected gas engines show that in TWC mode, in which selling the excess electricity is allowed, the prime mover works at full load condition during the year. In OWC mode, the prime mover follows the electricity demand profile and produces as much electricity as required. In HDF mode, a large amount of electricity should be bought due to the fact that to produce as much less heat as possible the prime mover works in low load conditions.

It is noted that because of high price of diesel fuel, in TWC mode of operation the diesel engine follows the electricity demand profile and there is no excess electricity to be sold. Therefore, the values of NAC and consequently the operational strategy results are the same for TWC and OWC modes. Similar to gas engine, the prime mover works in low load condition in HDF mode.

\section{Concluding remarks}

In this paper, a thermo-economic method for the optimum sizing and planning the operational strategy of internal combustion engines in a medium scale combined heat and power system is presented introducing the Net Annual Cost (NAC) criterion. The methodology has been adopted for an operational case study and the proposed method can be used for all types of prime movers as well as various sizes of the system.

\section{References}

[1] D. G. Newnan, T. G. Eschenbach, J. P. Lavelle, Engineering Economic Analysis, Oxford University Press, $9^{\text {th }}$ Edition, 2004.

[2] www.uow.edu.au/about/environment/energy/ planning

[3] www.integral.com.au

[4] www.originenergy.com.au

[5] www.aip.com.au

[6] J. Humphreys, Exploring a Carbon Tax for Australia, The Centre for Independent Studies, 2007, Available from: http://www.cis.org.au. 